\title{
Multiple intracranial vascular anomalies in a patient with stroke - a case report and short review of literature
}

\author{
Alexandru Dimancea ${ }^{1}$, Amalia Ene ${ }^{1,2}$, Bogdan Dorobat ${ }^{3}$, Andrei Simonov³ \\ Alexandra Carp ${ }^{3}$, Ovidiu Bajenaru ${ }^{1,2}$ \\ ${ }^{1}$ Neurology Department, University Emergency Hospital, Bucharest, Romania \\ 2"Carol Davila" University of Medicine and Pharmacy, Bucharest, Romania \\ ${ }^{3}$ Radiology Department, University Emergency Hospital, Bucharest, Romania
}

\begin{abstract}
Introduction. Non-saccular aneurysms are independent vascular entities, characterized by particular pathogenesis, localization, natural history and treatment. The fusiform subtype comprises an estimated tenth of all intracranial aneurysm, while the dolichoectatic subtype has different reported prevalence values, owing to several proposed diagnostic criteria (Smoker et al. criteria1 being the most widely used). The most frequent initial clinical presentations are ischemic stroke and transient ischemic attack, due to different hemodynamics and mechanism of formation. Prognosis is usually poor and progression can be demonstrated in almost half of ectatic vessels. Treatment can be either conservative in asymptomatic or ischemic presentations or by endovascular or microsurgical conduit in other cases. Case presentation. We report the case of a hypertensive 63-year old male patient with multiple intracranial vascular anomalies; he was initially admitted for ischemic stroke, with right hemiplegia and mixt aphasia. Non-contrast CT demonstrated a thrombosed left posterior cerebral artery aneurysm and contrast enhanced CT revealed a middle cerebral artery saccular aneurysm, two saccular and fusiform right PCA aneurysms and vertebrobasilar dolichoectasia. Cerebral angiography confirmed the previous findings and revealed lack of contrast at the level of the left PCA in the P2 segment. The patient was treated conservatively with aspirin and high-dose statins and had a favorable outcome, regaining part of his right-side function, object-naming and simple sentence construction capabilities.

Conclusions. Upon ischemic presentation in the case of non-saccular aneurysms, a conservative attitude is recommended. These aneurysms commonly have an unfavorable prognosis, demanding periodic follow-up for detecting recurrent ischemic events, possible hemorrhage and progression.
\end{abstract}

Keywords: non-saccular aneurysms, fusiform, basilar dolichoectasia, posterior cerebral artery aneurysm, case report

\section{INTRODUCTION}

Non-saccular intracranial aneurysms are independent vascular entities, set apart from their saccular counterparts by different pathogenesis, localization, natural history and potential treatments. Flemming et al. first classified non-saccular aneurysms into 3 separate groups: fusiform aneurysms (FA), dolichoectatic aneurysms (DEA) and transitional aneurysms (TA) (1).

Fusiform aneurysms are defined by a diameter of 1.5 times that of the parent vessel, without an imagistically demonstrated neck, involving a portion of an arterial segment (1). Etiological correla- tions have been demonstrated with dissection, atherosclerosis, collagen and elastin metabolism disorders and infection (2). In a study by Day et al. (3) regarding middle cerebral artery (MCA) FA, a sequence of aneurysm formation and evolution has been imagined, with a central role residing in intramural dissection (3). FA comprise $3-13 \%$ of all intracranial aneurysms (2). The vertebrobasilar (VB) system has a predisposition for developing FA (1).

Dolichoectasia (DE) or dolichoectatic aneurysms are defined by uniformly dilated arteries associated with marked elongation and tortuosity $(1,4)$. In a review of literature on DE, Gutierrez et al. identified the posterior circulation and particu- 
larly the basilar artery (BA) as the most common site for the dolichoectatic process $(55.5 \%$ of all cited cases affecting only the VB system) (4). Reported prevalence for DE is between 0.13 and $18 \%$ (4). Currently there is no imagistic gold standard for diagnosing DE; however, Smoker et al. (5) has suggested a series of criteria for basilar artery DE (BADE), widely utilized, pertaining to diameter, height of bifurcation and laterality compared to the clivus (5).

TA, the third variety of non-saccular aneurysms (1), refer to a superimposed focal dilatation on a previously ectatic vascular segment.

In a classification by Mizutani et al. regarding fusiform and dissecting aneurysms, FA are characterized by localized fragmented internal elastic lamina (IEL) and intimal thickening (6). Similarly, DE vessels present a disrupted IEL and atrophy of the muscle layer, widely believed to be a maladaptive vascular remodeling response to chronic arterial hypertension $(4,7,8)$. FA and DE are associated with concomitant abdominal aortic aneurysm or ectasia and intracranial saccular aneurysms $(1,8,9)$, which suggests a possible common pathogenesis.

Cerebral infarction and transient ischemic attack (TIA) are the most common initial symptomatology (27-40.4\%), followed by compressive symptoms and subarachnoid hemorrhage $(1,10)$.
Treatment for non-saccular aneurysms varies: if asymptomatic or in the case of initial ischemic presentation, conservative treatment is elected $(2,3)$. Otherwise, an open microsurgical conduit might be employed (clipping wrapping, bypass $(2,4)$ ), along with endovascular techniques (coiling, parent vessel occlusion, flow-diversion $(11,12)$.

In a recent meta-analysis on vertebrobasilar FA and DEA, natural history comprised a poor prognosis (10). Globally, non-saccular aneurysms progressed over time in nearly half the cases studied $(1,13)$. The most frequent cause of mortality is ischemic stroke $(1,10)$, with a high recurrence rate $(1,10,13)$.

Encompassing most of the described vascular anomalies, we report a case of an aged male patient presenting with a cerebral ischemic event. This case report was prepared following the CARE guidelines (14).

\section{CASE REPORT}

We present the case of a 63-year-old male patient, with a history of arterial hypertension and dyslipidemia, who was admitted to our clinic for acute ischemic stroke. Neurologic examination revealed right-sided hemiparesis, right central facial palsy, mixt aphasia and an up-going plantar reflex
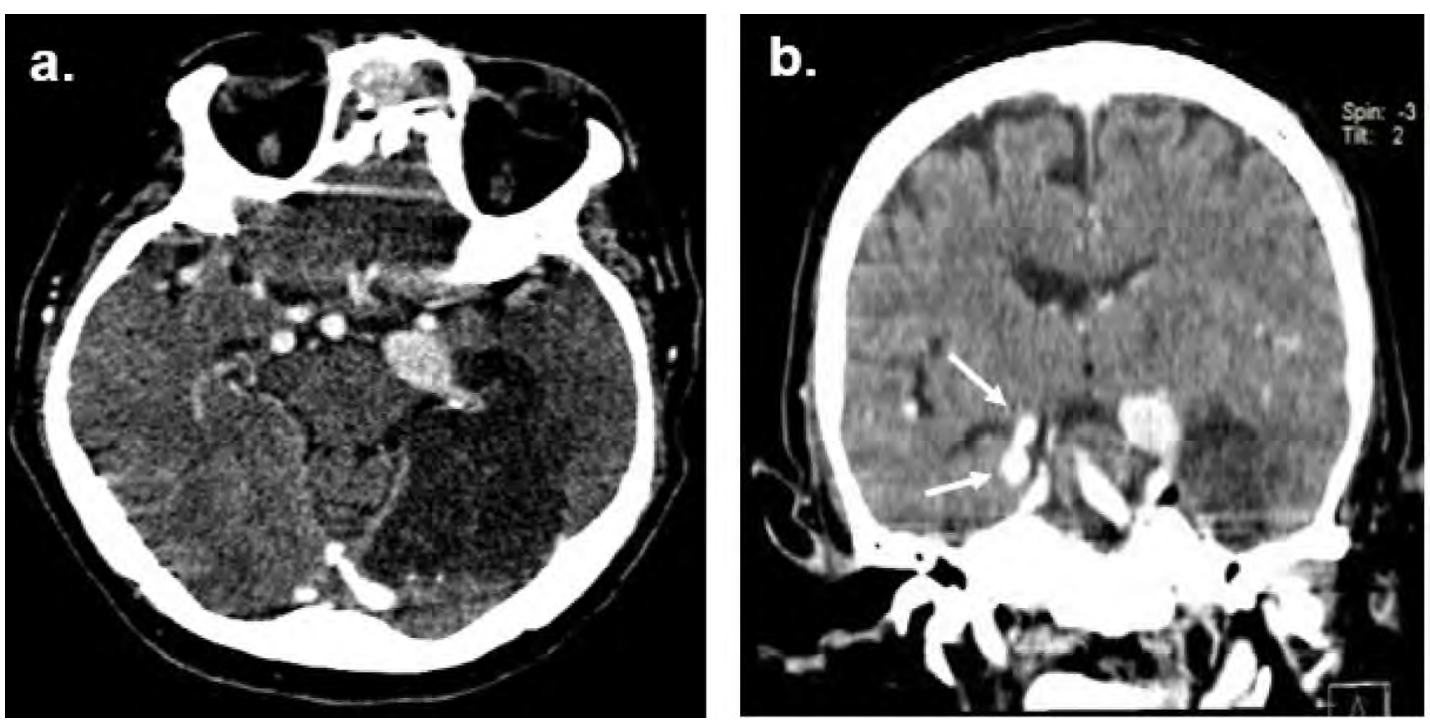

FIGURE 1. a. 1-week follow-up contrast-enhanced CT scan, axial view: left spontaneously hyperdense PCA fusiform aneurysm measured at $1.22 \mathrm{~cm}$ by $1.94 \mathrm{~cm}$ transverse and longitudinal diameters, respectively, with occipito-parahypocampal area of infarction. $b$. Contrast enhanced CT scan, coronal reconstruction, revealing multiple vascular anomalies: thrombosed left PCA aneurysms, two right PCA aneurysms (saccular and fusiform, respectively, with $8.9 \mathrm{~mm}$ and $7.1 \mathrm{~mm}$ respective maximum transverse diameters, white arrows) and a tortuous, elongated and dilated basilar artery. 

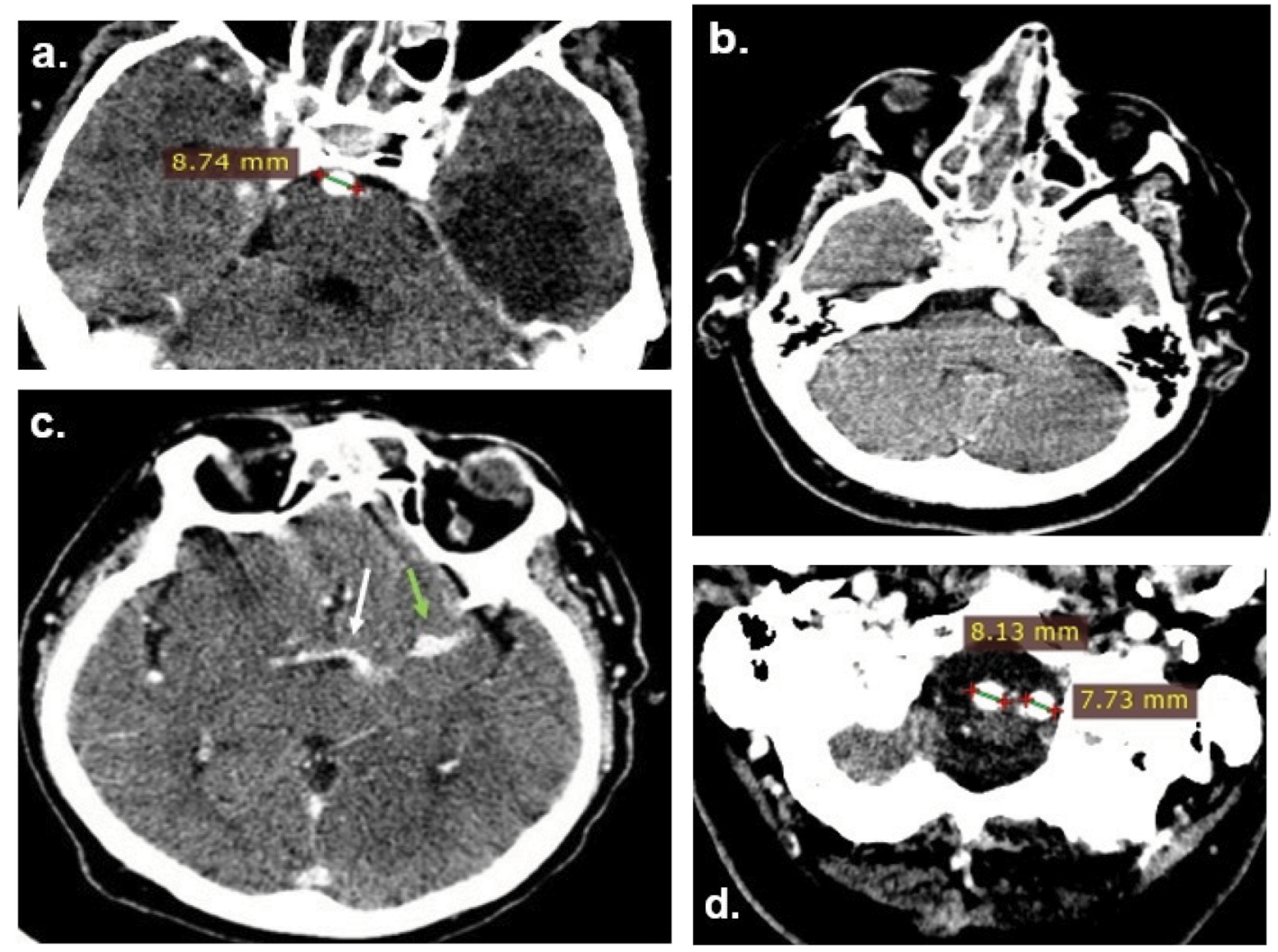

FIGURE 2. Application of Smoker's criteria on follow-up contrast enhanced CT scan: a. Basilar artery diameter at mid-pons level of $8.74 \mathrm{~mm}$; $b$. Height of BA bifurcation at the level of the 3rd ventricle floor (white arrow); c. BA course at the margin of the clivus; also, an M1 MCA aneurysm with a maximum diameter of $7.7 \mathrm{~mm}$ is revealed (green arrow); $d$. Vertebral arteries maximum diameters measured at $8.15 \mathrm{~mm}$ (right) and $7.27 \mathrm{~mm}$ (left).

on the right. Hospital presentation was under 2 hours from the debut of symptoms.

The emergency CT scan (not shown) revealed a spontaneously hyperdense, fusiform aneurysm at the level of the left posterior cerebral artery (PCA) $(1.22 \mathrm{~cm}$ by $1.94 \mathrm{~cm}$ transverse and longitudinal diameters) (Fig.1a); contrast enhanced-CT revealed another 2 aneurysms at the level of the right PCA (Fig 1b) and one at the level of the MCA. Intravenous alteplase administration was therefore not employed. Ultrasonography of cervico-cerebral vessels described normal flow speeds in all arteries and several atherosclerotic and calcified plaques at the level of common carotid artery bifurcation and internal carotid artery origin, on the right side.

One-week follow-up CT scan revealed an ischemic, subacute hypodensity affecting the left occipito-parahypocampal and thalamic regions, consistent with an ischemic stroke in the deep left PCA territory (Fig. 1).

Furthermore, based on Smoker et al. criteria (5) (Table 1), we established the diagnosis of VBDE
(Fig. 2), in addition to the aneurysms previously identified.

TABLE 1. Smoker et al. criteria (5)

\begin{tabular}{|l|l|}
\hline \multicolumn{2}{|c|}{ BA diameter $>4.5 \mathrm{~mm}$ at the level of midpons } \\
\hline $\begin{array}{l}\text { Laterality } \\
\text { of BA }\end{array}$ & $\begin{array}{l}\text { 1. Midline throughout } \\
\text { 2. Medial to the lateral margin of the clivus } \\
\text { 3. Lateral to the lateral margin of the clivus } \\
\text { 4. At the level of the cerebello-pontine angle }\end{array}$ \\
\hline & $\begin{array}{l}\text { 0. At or below the dorsum sellae } \\
\text { Height of BA } \\
\text { bifurcation }\end{array}$ \\
$\begin{array}{l}\text { 2. At the } 3^{\text {rd }} \text { ventricle floor } \\
\text { 3. Indenting and elevating the floor of the } 3^{\text {rd }} \\
\text { ventricle }\end{array}$ \\
\hline
\end{tabular}

Cerebral angiography (Fig. 3) revealed two aneurysmal dilatations at the level of the P2 segment of the right PCA, along with VBDE and absence of contrast at the level of the left PCA (thrombosed aneurysm). Furthermore, the left common carotid artery stemmed from the innominate artery (not shown).

The thrombosed aneurysm was not amenable to interventional treatment, nor did the other vascular 


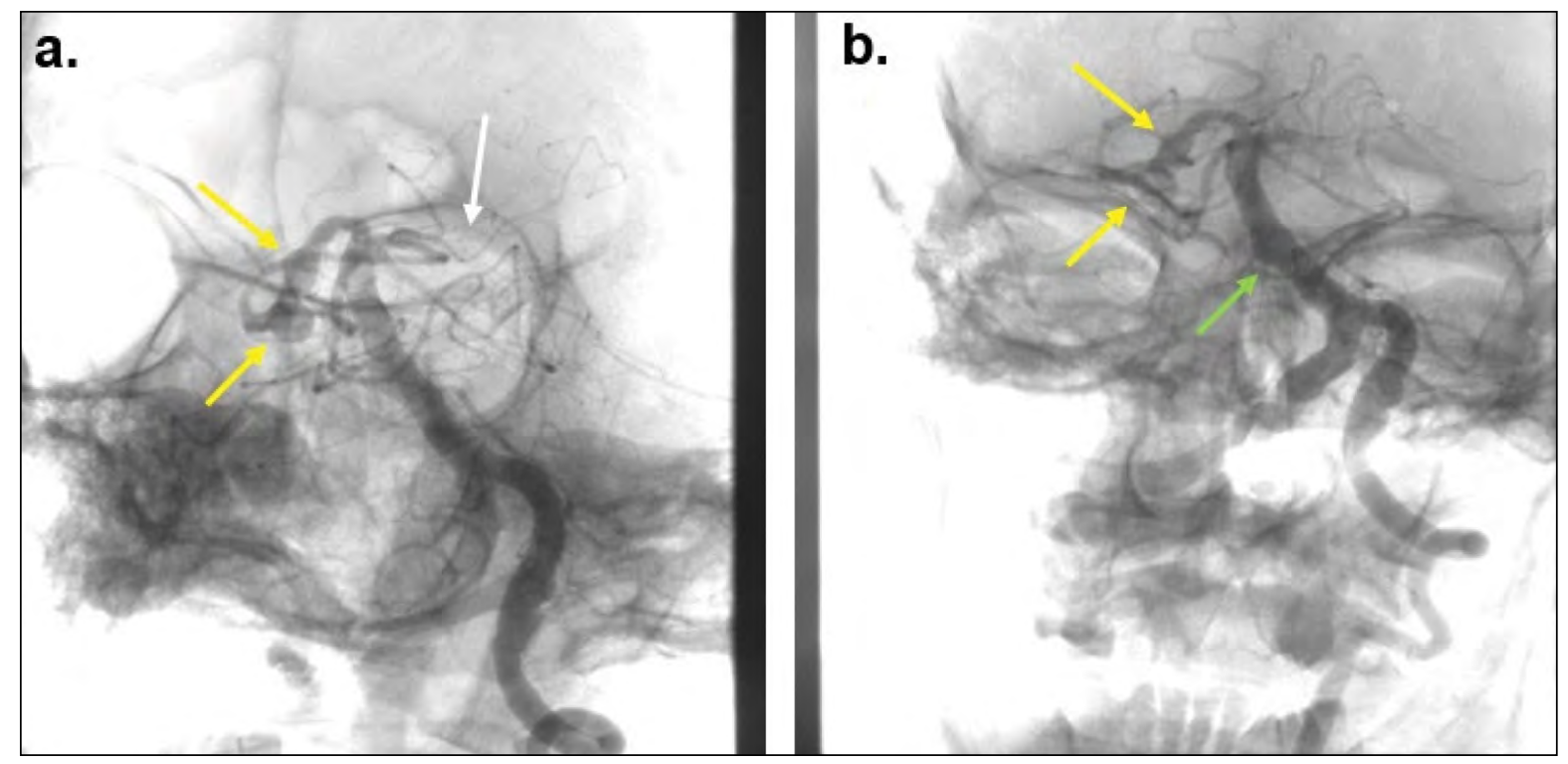

FIGURE 3. Angiography of the VB system: oblique (a.) and anteroposterior (b.) views, showing VBDE, two (fusiform and saccular) right PCA aneurysms (yellow arrows), lack of contrast at the level of the left PCA $P 2$ segment (white arrow); in addition, a probable transitional aneurysm can be observed at the level of the $B A$ (green arrow).

anomalies present an indication of interventional therapy. Therefore, the patient received conservative treatment with anti-platelet medication and high-dose statin. The patient's condition gradually improved and he partially regained the mobility of his right upper and lower limbs, named objects, formulated simple sentences and stood upright by his own at bedside. Upon discharge he was included in a neuro-rehabilitation program and clinical and imagistic follow-up plan.

\section{DISCUSSION}

Our case revealed multiple vascular anomalies in a male patient presenting with ischemic stroke. Concerning DE, the diagnosis was based on the aforementioned Smoker et al. criteria (5). In the published literature the application of these criteria resulted in a prevalence of $7.7-17 \%$ in series of stroke patients (7). More recently, in a multiethnic cohort of 718 stroke-free patients, the authors proposed a novel method for diagnosing intracranial artery DE (IADE): total cranial-volume adjusted artery diameter (9). Resulting prevalence was $4.7 \%$ for BADE (vs. $15.5 \%$ by using the previous criteria, with a cutoff used for defining BADE of 5.1 $\mathrm{mm})$.

Concerning the posterior circulation, PCA aneurysms are rare, with an estimated prevalence of $1 \%$ of all intracranial aneurysms $(3,15,16)$; this, cor- roborated with a reduced prevalence of the fusiform subtype resulted in several cases of PCA FA being documented $(3,15)$.

Multiple literature reviews have demonstrated a constant correlation of non-saccular aneurysms with several risk factors: old age, male gender and arterial hypertension. In addition, a statistical link has been identified between IADE and cerebral small vessel disease and other ectatic vascular anomalies. In a recent meta-analysis including 877 patients with VB non-saccular aneurysms, mean age was between $55-75$ years, $72 \%$ were male participants and $67 \%$ had arterial hypertension (10). In a review by Gutierrez et al. of 14 studies on VBDE (4), mean age was 63 years, $64 \%$ were male and $66 \%$ were hypertensive.

In a review by Brutto et al., patients with stroke and IADE had lacunar infarctions, severe leukoaraiosis and microbleeds in $36-42 \%$ of cases compared to patients with stroke, but without IADE (17-19\%) (7). In a series by Flemming et al., 18\% of patients with non-saccular aneurysms had an abdominal aortic aneurysms and $10 \%$ had concomitant intracranial saccular aneurysms (1). In 22 cases of PCA aneurysms, Beute et al. identified 12 cases with concomitant intracranial vascular anomalies (15). These associations suggest a common pathogenetic pathway, with consequences on different vascular territories. Our patient presented all the previously mentioned risk factors, as well as small 
vessel disease and two saccular aneurysms. However, he was not further investigated concerning a potential aortic abnormality.

Our patient presented with an initial ischemic event, caused by left PCA aneurysm thrombosis. In a study by Flemming et al. (1), ischemic stroke and transient ischemic attack were the most frequent clinical presentations (27\%), followed by compressive symptoms (22\%); however, most non-saccular aneurysms (DE in particular) were incidental imagistic findings (1). Ischemic symptoms were the initial presentation in almost $31 \%$ of FA in the series by Day et al. (3) and remained the major initial symptomatology in a recent meta-analysis (40.4\%) (10). Day et al. (3) proposed a process consisting of several steps leading to spontaneous FA formation. Pathogenesis revolves around intramural hemorrhage following arterial dissection. The authors considered subintimal dissection as being responsible for the progressive luminal narrowing altering local hemodynamics and creating a thrombogenic milieu.

VB non-saccular aneurysms have, in general, a poor natural history $(1,10)$. In a series of 159 patients, almost one third of deaths were related to ischemic events. The 1-, 5- and 10-year risk of ischemic stroke after diagnosis was $2.7 \%, 11.3 \%$ and $15.9 \%$, respectively. Predictors of cerebral ischemia due to VB non-saccular aneurysms were symptomatic aneurysms at presentation, TA and prior ischemic events. Nearly half of the cases studied demonstrated diameter growth at follow-up (1).

In a series of 156 patients with DE by Passero and Rossi et al. (13), with a mean follow-up of 11.7 years, VBDE progressed in $43 \%$ of the patients. Cumulative risk of a first recurrent stroke was $56 \%$ at 10 years and $79 \%$ at 15 years. Stroke was the most common cause of death, accounting for $40 \%$ of the deaths in this series.

In a recent meta-analysis (10), the authors considered FA and DEA as separate vascular entities, due to different natural history: FA had a superior rupture rate (3\%/year vs. almost no risk) and a superior growth rate $(12 \% /$ year vs. $3 \% /$ year for DEA).

Taking into account all concomitant anomalies, our patient mandates a thorough clinical and imagistic follow-up, focusing on both cerebral and extra-cerebral sites.
In our patient treatment was conservative with anti-platelet agent, statin and efficient control of blood pressure; this was due to an initial ischemic event and to the lack of symptomatology concerning the other vascular incidental findings. Intravenous infusion of alteplase was not attempted owing to the multiple concomitant intracranial vascular anomalies. In 22 cases of FA treated by Park et al. with different therapies (wrapping, clipping, resection, proximal occlusion, bypass and combinations of these), the authors concluded that asymptomatic aneurysms as well as stenotic or occluding lesions should be treated conservatively (2). However, in a review on $\mathrm{DE}$, the authors conclude that there is no specific treatment and no controlled trial attesting efficacy of either aspirin or warfarin (4).

In a series of 102 MCAFA (3), the authors advocate for maximal interventional treatment (occlusion of parent vessel with trapping of the aneurysm, resection and vascular bypass). Moreover, endovascular therapies are increasingly utilized, with Ciceri et al. and Taqi et al. successfully treating a small series of mostly saccular PCA aneurysms by means of endovascular coiling $(11,16)$; the efficiency of these methods was also confirmed in a review by Awad et al. (12) for posterior circulation FA (proposing flow-diversion in addition to stenting and coiling).

\section{CONCLUSIONS}

Non-saccular aneurysms present a different clinical behavior and challenge compared to saccular aneurysms, frequently leading to ischemic events. Association of VBDE and FA with concomitant saccular aneurysms may orient towards a common vessel wall pathogenetic mechanism within different territories, mandating thorough imagistic investigation (cerebral and aortic). Owing to a poor natural history of non-saccular aneurysms, patients require periodic clinical and imagistic follow-up.

\section{Consent}

Signed consent for the publishing of the patient's clinical and imagistic data was obtained from a close relative. 


\section{REFERENCES}

1. Flemming KD, Wiebers $\mathrm{DO}$, Brown RD et al. The natural history of radiographically defined vertebrobasilar nonsaccular intracranial aneurysms. Cerebrovasc Dis. 2005;20(4):270-279. doi:10.1159/000087710

2. Park S-H, Yim M-B, Lee C-Y, Kim E, Son E-I. Intracranial Fusiform Aneurysms: It's Pathogenesis, Clinical Characteristics and Managements. J Korean Neurosurg Soc. 2008;44(3):116. doi:10.3340/jkns.2008.44.3.116

3. Day AL, Gaposchkin CG, Yu CJ, Rivet DJ, Dacey RG. Spontaneous fusiform middle cerebral artery aneurysms: characteristics and a proposed mechanism of formation. J Neurosurg. 2003;99(2):228240. doi:10.3171/jns.2003.99.2.0228

4. Gutierrez J, Sacco RL, Wright CB. Dolichoectasiag-an evolving arterial disease. Nat Rev Neurol. 2011;7(1):41-50. doi:10.1038/ nrneurol.2010.181

5. Smoker WRK, Price MJ, Keyes WD, Corbett JJ, Gentry LR. High-resolution computed tomography of the basilar artery: I. Normal size and position. Am J Neuroradiol. 1986;7(1):55-60.

6. Mizutani T, Miki Y, Kojima H, Suzuki H. Proposed Classification of Nonatherosclerotic Cerebral Fusiform and Dissecting Aneurysms. Neurosurgery. 1999;45(2):253-259. doi:10.1097/00006123199908000-00010

7. Del Brutto VJ, Ortiz JG, Biller J. Intracranial arterial dolichoectasia. Front Neurol. 2017;8(JUL):12-14. doi:10.3389/fneur.2017.00344

8. Rouchaud A, Cloft HJ, Nasr DM et al. Clinical and Imaging Characteristics of Diffuse Intracranial Dolichoectasia. Am J Neuroradiol. 2017;38(5):915-922. doi:10.3174/ajnr.a5102
9. Gutierrez J. Dolichoectasia Diagnostic Methods in a Multi-Ethnic, Stroke-Free Cohort: Results from the Northern Manhattan Study. J Neuroimaging. 2015;24(3):226-231.

doi:10.1111/j.1552-6569.2012.00781.x.Dolichoectasia

10. Nasr DM, Flemming KD, Lanzino $G$ et al. Natural History of Vertebrobasilar Dolichoectatic and Fusiform Aneurysms: A Systematic Review and Meta-Analysis. Cerebrovasc Dis. 2018;45(1-2):68-77. doi:10.1159/000486866

11. Taqi MA, Lazzaro MA, Pandya DJ, Badruddin A, Zaidat OO. Dissecting aneurysms of posterior cerebral artery: Clinical presentation, angiographic findings, treatment, and outcome. Front Neurol. 2011; JUN(June):1-6. doi:10.3389/fneur.2011.00038

12. Awad AJ, Mascitelli JR, Haroun RR, De Leacy RA, Fifi JT, Mocco J. Endovascular management of fusiform aneurysms in the posterior circulation: the era of flow diversion. Neurosurg Focus. 2017; 42(6):E14. doi:10.3171/2017.3.focus 1748

13. Passero SG, Rossi S. Natural history of vertebrobasilar dolichoectasia. Neurology. 2008;70(1):66-72. doi:10.1212/01. wnl.0000286947.89193.f3

14. Riley DS, Barber MS, Kienle GS et al. CARE guidelines for case reports: explanation and elaboration document. J Clin Epidemiol. 2017; 89:218-235. doi:10.1016/j.jclinepi.2017.04.026

15. Beute GN. Endovascular Treatment of Posterior Cerebral. Ajnr. 2006.

16. Ciceri EF, Klucznik RP, Grossman RG, Rose JE, Mawad ME. Aneurysms of the Posterior Cerebral Artery: Classification and Endovascular Treatment Elisa. 2001;AJNR Am J(January):1-8. papers://4f789145-6e78-4c33-b1af-ed123c0287ec/Paper/p152. 